\title{
A Method of Measuring Transient Plume Properties
}

\author{
Robert B. Lobbia* and Alec D. Gallimore ${ }^{\dagger}$
}

The University of Michigan, Ann Arbor, MI, 48109

In an effort to study the transient fluctuations of plasma properties downstream of a Hall Effect Thruster (HET) on a microsecond timescale, a high-speed Langmuir probe measurement system is developed and tested. A high-voltage $( \pm 225 \mathrm{~V})$, broadband (DC $-2 \mathrm{MHz}) 30-\mathrm{W}$ power amplifier is constructed to drive the Langmuir probe voltage sweep signal, and high-voltage current measurement circuitry is used to measure the plasma current drawn in the HET plume. A null probe, not in contact with plasma, is driven simultaneously with the Langmuir probe to measure and thereby correct for leakage currents, which nominally measure $5 \mathrm{~mA}$. The power amplifier sources or sinks up to $200 \mathrm{~mA}$, while the current measurement resolution is $3 \mu \mathrm{A}$ (with $\mathrm{S} / \mathrm{N}>\mathbf{8 0} \mathrm{dB}$ ). Rapid successive voltage sweeping enables the acquisition of the plume's transient nature in terms of the variation of the plume plasma density, electron temperature, and floating and plasma potentials. After automated processing of the Langmuir probe I-V traces, plasma properties are recorded at intervals of 10 microseconds, thereby allowing for the examination of the highly turbulent plasma flow from the HET. While various plasma oscillations are known to exist above the frequency of $50 \mathrm{kHz}$ for a HET, the largest amplitude fluctuations, caused by the thruster "breathing mode," are indeed captured and related to the measured discharge current oscillations. Data acquisition (DAQ) and control are provided by a multichannel 2 MHz 16-bit dedicated DAQ system, interface PC, and a motion control PC (all systems networked together for synchronous data collection). A grid in the plume of the HET measuring $31 \mathrm{~cm}$ by $50 \mathrm{~cm}$ is examined, collecting a total of 352 datasets. To characterize and quantify the measured turbulent plasma transient fluctuations, Fourier transforms are applied to large data sets for post processing in the frequency domain, thus constructing various Bode plots and power spectra representative of the behavior of the plasma across frequencies from DC to $50 \mathrm{kHz}$. Transfer functions are used to model the thrusterplume system from the measured plasma properties and discharge current signals. Fourier synthesis of these transfer functions is then used to create time-coherent 2D maps of the transient plume properties in the form of contour map movies with a $10 \mu$ s framerate - providing dramatic visualizations of the thruster breathing mode at $17.8 \mathrm{kHz}$. A 600-W Busek HET (BHT-600) is operated at a 200-V 2-A discharge in the Large Vacuum Test Facility to create the plume studied in these experiments.

\section{Nomenclature}

$=$ Discrete Fourier transform coefficient

$=$ Stray Capacitance $[F]$

$=$ Electron charge constant [Coulombs]

$=$ Frequency $[\mathrm{Hz}]$

$=$ Discrete Transfer Function between two dynamic signals

$=$ Dynamic discharge current of Hall thruster [A]

$=$ Total specific impulse $[\mathrm{sec}]$

$=$ Boltzmann's constant $[\mathrm{J} / \mathrm{K}]$

$=$ Electron mass $[\mathrm{kg}]$

$=$ Ion mass $[\mathrm{kg}]$

$=$ Number of sets to average

$=$ Number of distinct sample points

$=$ Total Transient electron density $\left[\mathrm{m}^{-3}\right]$

$=$ Transient fluctuation component of electron density $\left[\mathrm{m}^{-3}\right]$

$=$ Discharge voltage efficiency

$=$ Transient electron Temperature $[\mathrm{eV}]$

$=$ Discharge voltage of Hall thruster $[\mathrm{V}]$

\footnotetext{
* PhD Candidate, Aerospace Engineering Dept., FXB Building Room 1052, AIAA Student Member.

${ }^{\dagger}$ Arthur F. Thurnau Professor, Aerospace Engineering Dept., Associate Fellow AIAA.
} 


$\begin{array}{ll}V_{f}(t) & =\text { Transient floating potential [V] } \\ V_{p}(t) & =\text { Transient plasma potential [V] } \\ \omega_{p, i} & =\text { Ion plasma frequency [Radians/sec] }\end{array}$

\section{Introduction}

$\mathrm{T}$ The dynamic electromagnetic nature of plasmas gives rise to a broad spectrum of observable instabilities: in the interest of electric propulsion devices this range is roughly $1 \mathrm{kHz}$ to $2 \mathrm{GHz}[1,2]$. The bulk of most prior electric propulsion research on plasma instabilities has focused on characterizing the magnitude and frequency characteristics [3-5] and suggesting theories for the physics creating the oscillations [2, 6, 7]. High-resolution, spatially and temporally resolved measurements of plasma property oscillations, including phase, is absent from the literature and the goal of this endeavor is to fill that gap; providing a detailed and completely time resolved picture of the full plume field created by the operation of a Hall effect thruster (HET). The stochastic turbulent nature of plasmas has placed a large emphasis in the development of and use of time-averaging plasma diagnostics to study HETs, Tokamaks, Helicons, and other plasma systems. While the time-averaged plasma properties acquired from these DC diagnostics result in smooth contour maps of electron density (see Figure 13), temperature, or other parameters, this view neglects the underling transient fluctuations that permeate through every plasma flow field. The troubling disconnect between experimental HET measurements and numerical simulations of HETs in terms of anomalous electron transport, performance matching, erosion, and other properties, may in part be due an emphasis on the DC view of the plume properties. Indeed, it is well known that HETs operate more like undamped harmonic oscillators [8] expunging axial waves of ions undergoing bursts of ionization and consequent acceleration. This process is referred to as the thruster "breathing mode" and it cycles at a rate in the range 10-60 kHz. Breathing mode oscillations have been observed for decades ever since the first Hall thrusters (e.g., the SPT-100) were operated. Generally, the breathing mode oscillations are measured by recording temporal evolution of the discharge current signal, but a handful of experiments have indicated that these oscillations are also present within the discharge channel and near field plume of a HET [4, 9, 10]. A Langmuir probe is perhaps the simplest plasma diagnostic available both in terms of theory and construction, making it a prime sensor to adapt for performing high-speed timeresolved measurements. Various attempts to perform high-speed Langmuir probe measurements by prior researchers [9, 11-17] have achieved limited success, often plagued by the adverse effects of stray capacitance, poor signal to noise ratios, small sample set sizes, or awkward non-physically correct windowed averaging. In this study, we have overcome these difficulties providing spatially detailed, low-noise, time-resolved plasma properties downstream from a HET.

\section{Experimental Setup}

\section{A. Large Vacuum Test Facility}

The LVTF is a cylindrical vacuum chamber measuring 6 meters in diameter and 9 meters in length. Four 400-CFM mechanical pumps and two 2,000-CFM blowers serve to evacuate the chamber to a rough vacuum pressure of 60 mtorr. A high-vacuum base pressure of $2 \times 10^{-6}$ torr is attained by operating four of the seven available liquid- $\mathrm{N}_{2}$ baffled CVI TM-1200 re-entrant cryopumps with an aggregate pumping speed of 120,000 liters/sec on xenon.

\section{B. Busek BHT-600 Hall Thruster}

A cluster of four BHT-600 Busek Hall effect thrusters is operated in single-thruster mode for the extent of this experiment. While the thruster power is adjustable, a fixed operation point of $400 \mathrm{~W}(200 \mathrm{~V}, 2 \mathrm{~A}, 2.6 \mathrm{mg} / \mathrm{sec}$ xenon discharge) is considered. The BHT-600 cluster is mounted in a fixed manner at Station 1 of the LVTF with the Langmuir probe and null probe mounted to a pair of linear positioning tables. Nominal performance of a single BHT-600 thruster (at 2

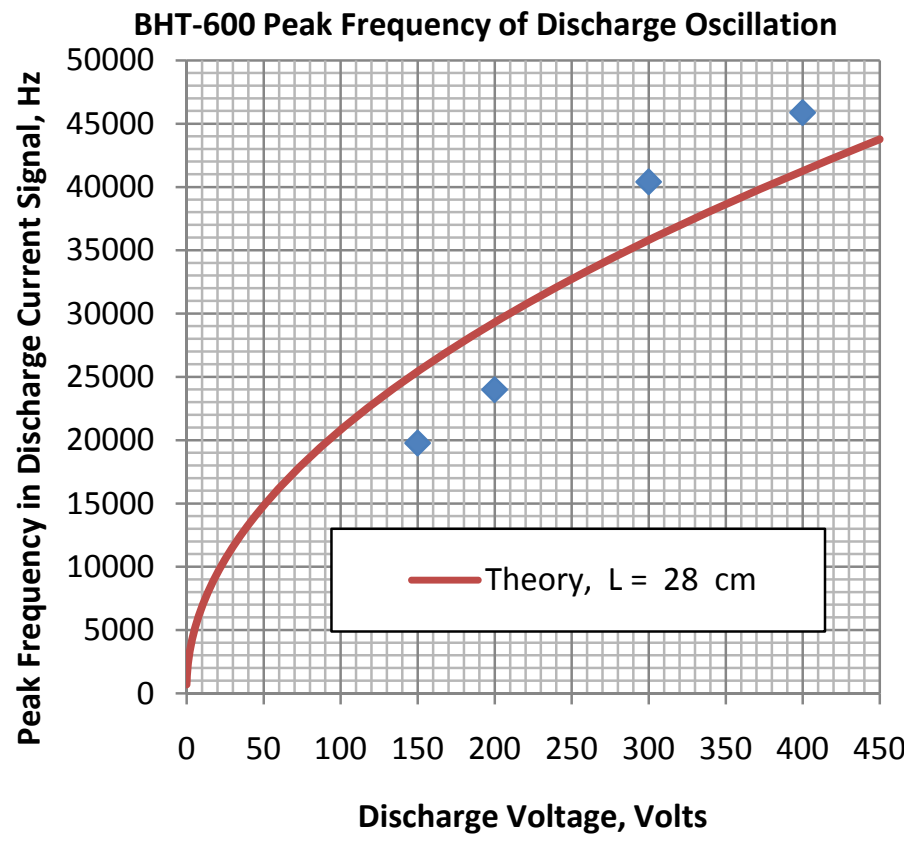

Figure 1. Variation of breathing mode as function of discharge voltage: experimental and theoretical scaling results for constant 600 $\mathrm{W}$ power operation of the BHT-600 thruster.

American Institute of Aeronautics and Astronautics

092407 
$300 \mathrm{~V}$ ) is rated at $I_{s p}=1530 \mathrm{sec}$, thrust efficiency $=49 \%$, thrust $=39.1 \mathrm{mN}$ [18]. Each thruster in the cluster operates using an independent set of five Sorenson power supplies (heater supply, keeper supply, inner and outer electromagnet supplies, and discharge supply) and cathode and anode mass flow controllers. This enables the operation of a single thruster, the full cluster, or any configuration in between. Prior to data collection the thruster is fired for a minimum of 30 minutes to warm up the thruster components and eliminate startup transients [19] - especially strong oscillations observed in the initial operation of a HET. At steady-state, oscillations still dominate the discharge current signal resultant from thruster breathing mode (ionization instability). Figure 1 displays the variation of the breathing mode for the BHT-600 at several discharge voltages along with a simple kinetic model of this variation.

\section{Langmuir Probe and Null Probe}

Langmuir probes are a simple plasma diagnostic used to estimate plasma properties such as electron temperature and electron/ion density. A typical Langmuir probe consists of an exposed metal surface, such as tungsten or nickel, of known surface area. In the operation of a Langmuir probe, the probe bias voltage is slowly swept across the voltage range of interest (e.g. - $100 \mathrm{~V}$ to $+100 \mathrm{~V}$ ) whilst measuring the current flow through a conducting wire attached to the probe. This procedure generates the characteristic I$\mathrm{V}$ (current-voltage) trace that is analyzed using thin sheath Langmuir probe theory (here, Debye length over probe radius, $\lambda_{D} / r_{p} \approx 10$ ) to extract an estimate of the electron temperature and plasma density along with other parameters of interest.

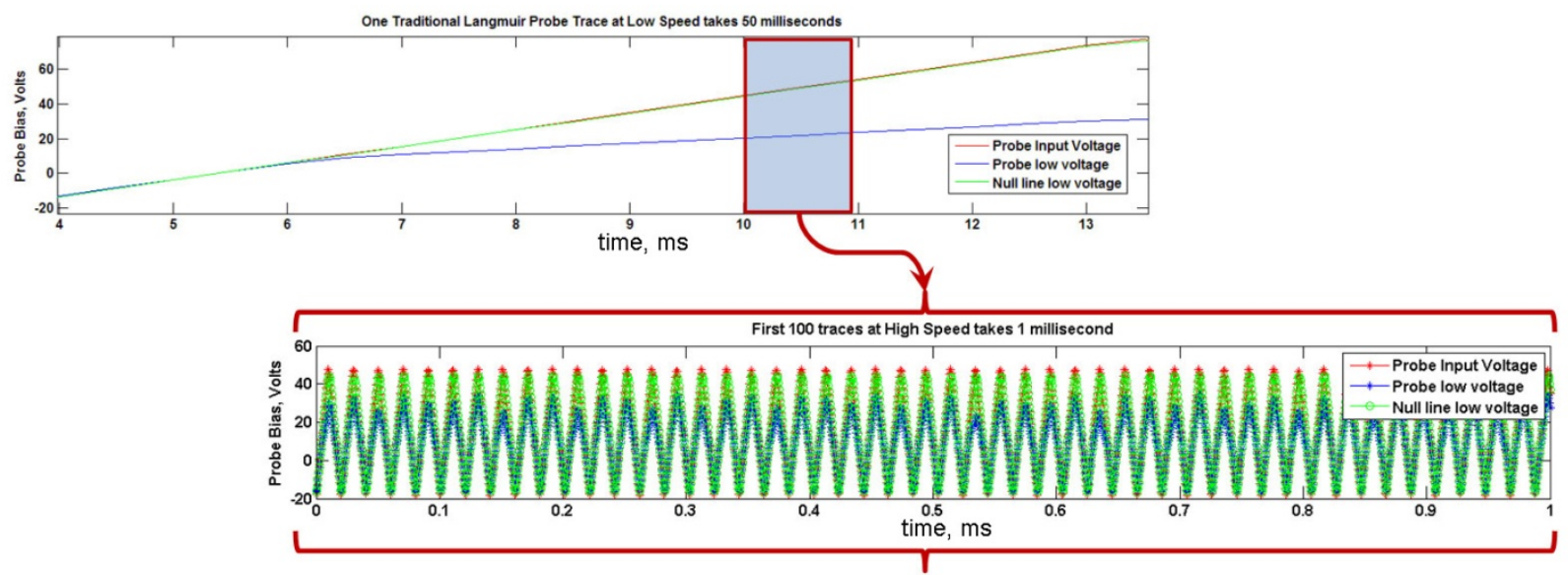

Figure 2. Upper plot, traditional "slow" Langmuir probe voltage signal (raw data) versus lower plot, high-speed Langmuir probe voltage signal. Data are taken with both methods and mean results matched within expected error bounds.

In order to measure transient plasma properties, one may sweep the probe bias rapidly enough that individual I-V traces are generated at least twice the rate at which the transient oscillations of interest are occurring. For this experiment we choose to sweep our probe bias every $10 \mu \mathrm{s}(100 \mathrm{kHz})$ allowing us to observe transient phenomena in the frequency range of DC to $50 \mathrm{kHz}$. Langmuir probe analysis is still valid when rapidly sweeping the probe bias so long as the sweep frequency remains below the characteristic sheath formation frequency $[12,20]$. The timescale of sheath formation must be small enough that the sheath is properly formed during a high-speed Langmuir probe bias sweep. The frequency associated with this timescale is approximately $300 \mathrm{kHz}$ (Equation 1), which places our probing at $100 \mathrm{kHz}$ below the theoretical limit.

$$
\text { Sheath formation timescale: } f_{\text {sheath }} \approx \frac{k T_{e}}{2 e V_{\text {sweep }}} \frac{\omega_{p, i}}{2 \pi}=\frac{5 \mathrm{eV}}{100 \mathrm{~V}} 6 \mathrm{MHz}=300 \mathrm{kHz} \quad \text { Equation } 1
$$

Operation of our high-speed Langmuir probe close to the theoretical limit may introduce some distortion in Langmuir probe characteristics due to incomplete ion sheath formation, but we expect these effects to be minimal and non-problematic in our analysis since we do not explicitly use the ion saturation portion of the I-V characteristic. The electron sheath formed at highpositive bias however, is critical in determining the electron saturation current - which is directly proportional to the electron density evaluated from the I-V characteristic. Fortunately, this electron sheath forms a factor of $\sqrt{m_{i} / m_{e}} \approx 500$ times faster, or at the characteristic rate of $150 \mathrm{MHz}$.

When the voltage of a conductor is quickly changed, a leakage current is induced from the nearest ground plane, as described by the basic capacitance relation: 


$$
I_{\text {leakage }}=C_{\text {stray }} \frac{d V}{d t}
$$

Equation 2

Every conductor has a finite but generally small measure of stray capacitance, $\mathrm{C}_{\text {stray }}$, that contributes to the generation of leakage current. A DC or steady voltage generates zero leakage current, thus tradition Langmuir probe experiments wherein the probe bias is slowly swept generate negligible leakage currents. As an example, a circuit of relatively small capacitance of $240 \mathrm{pF}$ (equivalent capacitance of $8 \mathrm{ft}$ of BNC cable or $20 \mathrm{ft}$ of insulated wire near a ground plane), sweeping from $-100 \mathrm{~V}$ to $+100 \mathrm{~V}$ in $10 \mu \mathrm{s}$, generates a leakage current of $I_{\text {leakage }}=\left(240 \times 10^{-12} \mathrm{~F}\right)(200 \mathrm{~V}) /\left(10 \times 10^{-6} \mu \mathrm{s}\right)=5 \mathrm{~mA}$. This amount of current could easily exceed the plasma current one is attempting to measure with a typical Langmuir probe. Therefore, a means of minimizing or correcting for this current is necessary. For this experiment, a specially designed transmission line is used to conduct the Langmuir probe signals with a theoretical capacitance of about $2.4 \mathrm{pF} / \mathrm{ft}$. A null probe is also positioned close (within $5 \mathrm{~mm}$ ) to the Langmuir probe, and is used to measure the instantaneous leakage current of the transmission line.

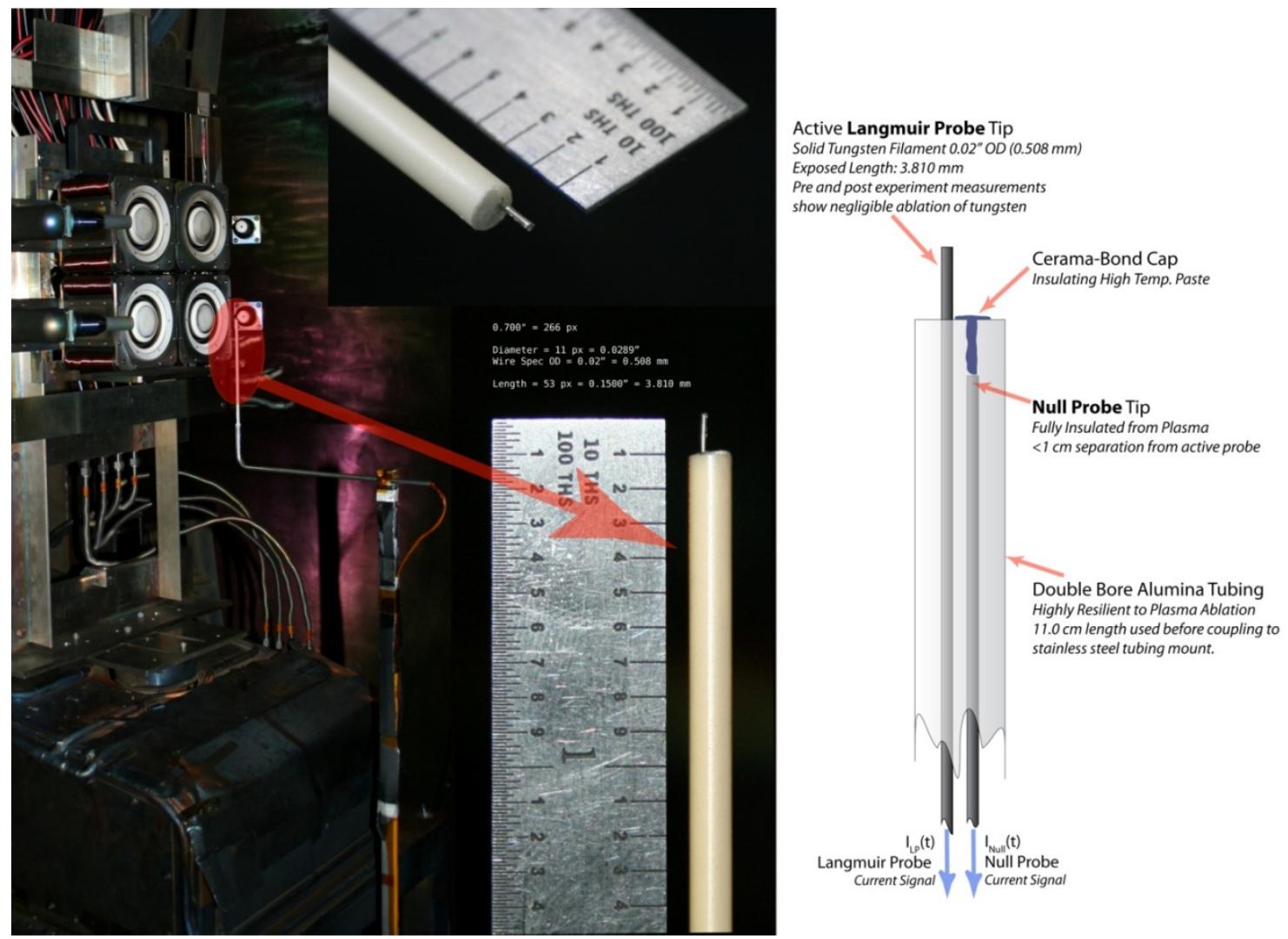

Figure 3. Far left photo shows Busek HET setup in LVTF with Langmuir probe about $10 \mathrm{~cm}$ downstream of lower right clustered thruster. Close-ups of Langmuir probe are also shown. On the right, a schematic of both probes is presented including the position of the null probe (not visible in photos due to complete ceramic and Alumina encapsulation).

The true plasma current from the Langmuir probe is obtained by directly subtracting this leakage current from the total current measured by the conductor connected to the Langmuir probe. Since the conductors transmitting the signals for the Langmuir probe and null probe are nearly identical and in close proximity, the direct subtraction provides a nearly exact correction.

The use of a null probe is a rather effective solution that mitigates
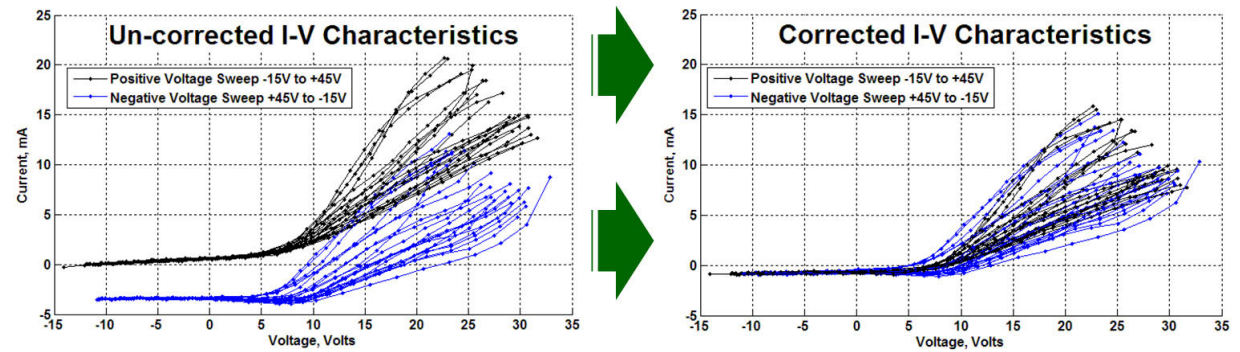

Figure 4. Using the null probe dynamic measurement of leakage current accurately corrects for stray capacitive effects.

American Institute of Aeronautics and Astronautics 092407 
the effects of stray capacitance that otherwise plague high-speed Langmuir probe measurements. The use of such a null probe was motivated by other experiments in which the stray capacitance was imperfectly estimated with either a length of wire [12, 16] or variable capacitor. A few examples of dummy probes [21] near the active probe exist but are operated at much lower frequencies; i.e., less than $1 \mathrm{kHz}$. Here, great effort was applied in creating nearly duplicate setups for the Langmuir and null probes, so as to create a very accurate capacitance match and allow for operation at frequencies $>100 \mathrm{kHz}$. Indeed, effects such as plasma dynamic capacitance are even corrected for, since the null probe is immersed (while fully insulated) in the same region of plasma as the Langmuir probe (see Figure 5).

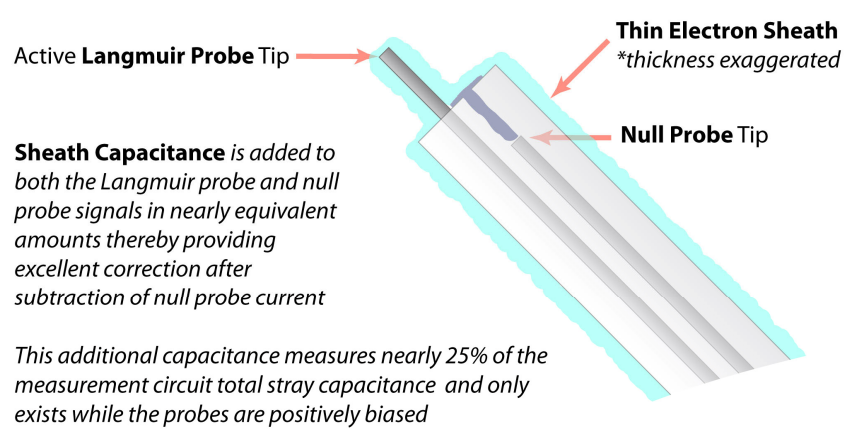

Figure 5. A thin electron sheath forms around both the Langmuir probe and null probe at high bias potential. The extra stray capacitance created by this transient sheath is well corrected for by the null probe. Actual sheath thickness or Debye length is approximately $0.05 \mathrm{~mm}$ for most data, considerably smaller than in above illustration.
An added benefit of the null probe is noise cancellation. Since shielded wires possess prohibitively large capacitance, they could not be used here, making EM noise pickup a valid concern. Since the transmission lines for the Langmuir and null probes are in close proximity at all points, we also subtract all EM signal noise picked up when we subtract the null current from the Langmuir probe current. For the Langmuir and null probes the current measurement occurs outside the vacuum chamber, and movement of this current sensing circuitry closer to the actual probes (inside of the vacuum chamber) would further reduce the stray capacitance. Yet the effectiveness of the null probe leakage current cancellation allowed for the circuitry to remain outside the vacuum where the shunts and circuitry are better cooled and monitored.

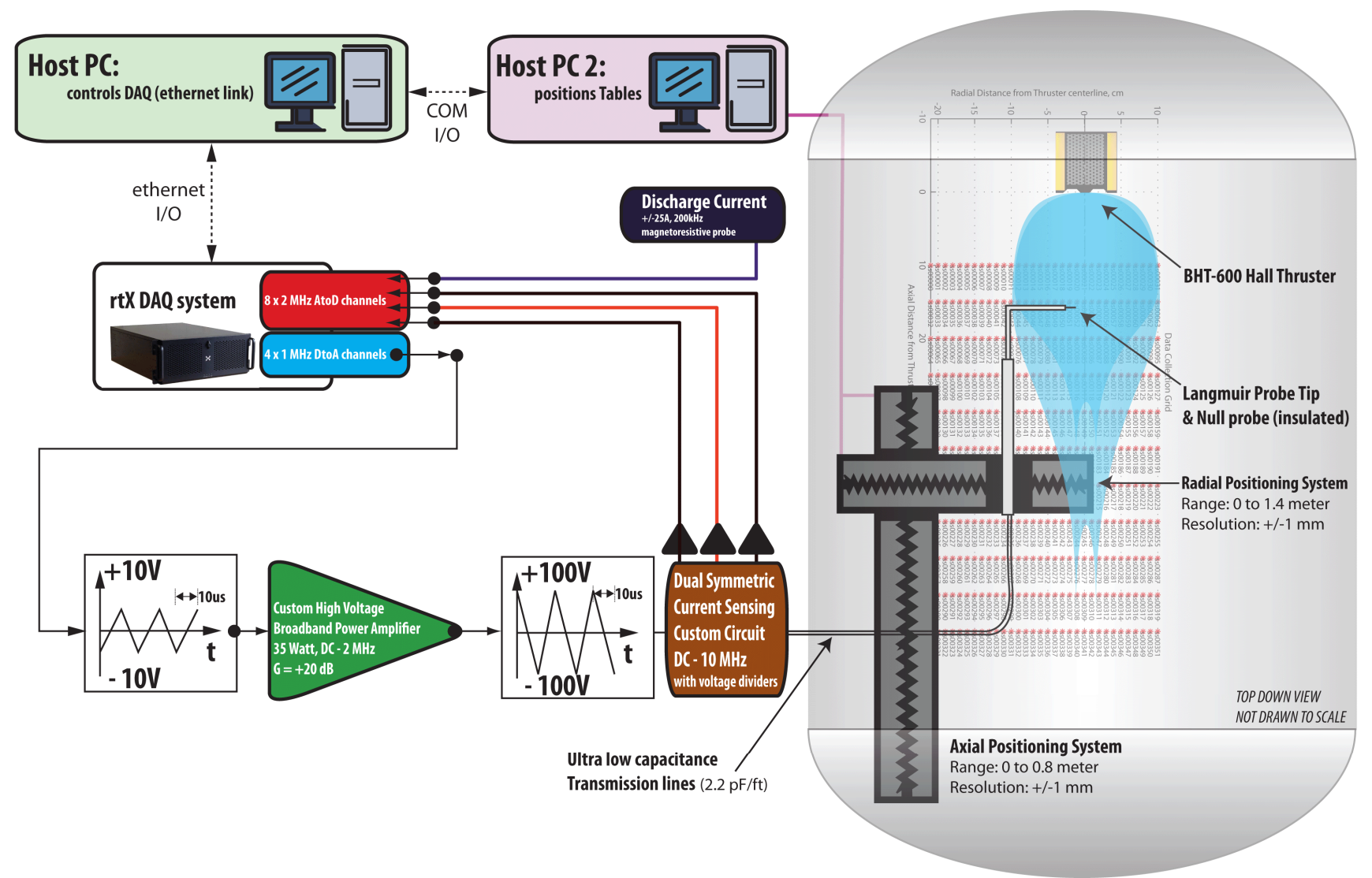

Figure 6. Experimental Configuration Schematic. Not drawn to scale. A host PC controls the dedicated high-speed DAQ system that drives and records the Langmuir probe data collection. High-speed broadband probes, amplifiers, and DAC/ADC, allow capture of plasma plume transients less than 10 microseconds. 


\section{Broadband Power Amplifier and Symmetric Current Measurement Custom Circuits}

One of the first challenges of this experiment was the lack of "commercially available off-the-shelf" (COTS) broadband highvoltage power amplifiers that could meet the bandwidth, voltage, and current requirements: DC $-1 \mathrm{MHz}, \pm 100 \mathrm{~V}, \pm 100 \mathrm{~mA}$. Additionally, there is a scarcity of COTS current sensors able to meet the requirements of this experiment: flat bandwidth from DC to $10 \mathrm{MHz}, 10-\mu \mathrm{A}$ resolution and $100 \mathrm{~mA}$ full scale, with high-voltage isolation of output signal. The few commercial probes that come close (namely, split-core Hall probes and high-voltage differential probes) provide insufficient signal-to-noise (S/N) ratios $<60 \mathrm{~dB}$. Thankfully, a solution was found in a new class of high-frequency actively cooled shunts together with a carefully laid out set of matched (symmetric) differential amplifiers with active voltage dividing networks. Due to the challenging design requirements for all this custom circuitry, SPICE (Simulation Program with Integrated Circuit Emphasis) was used to design the amplifier and a pair current sensors with specifications that would meet and ultimately exceed those required for this experiment. Including the specially designed transmission lines in the SPICE design process allowed for accurate performance simulations in terms of attaining stable and distortion free operation of the desired sawtooth and triangle waveforms typical to Langmuir probe bias sweeps. The effectiveness of the Null probe correction was also made clear in the SPICE simulations. In the construction of the circuits $\mathrm{E} / \mathrm{M}$ noise filtering and shielding (of power and signal lines), along with active cooling and temperature monitoring were included to ensure ultra-low noise $(\mathrm{S} / \mathrm{N}=80 \mathrm{~dB})$ and repeatable operation of the broadband amplifier and current measurement.

\section{Theory}

\section{E. Fourier Analysis}

The highly stochastic nature of plasma oscillations in the plume downstream from a Hall-effect thruster limits the usefulness of time series data. Consequently, we decided to use of frequency domain plots for data spectral analysis. The discrete Fourier transformation of an N-point time series signal $I_{d}(t)$ is defined as:

$$
\mathcal{F}\left\{I_{d}(\Delta t \cdot k)\right\}=A_{I_{d}}(k)=\sum_{j=0}^{N-1} I_{d}(\Delta t \cdot j) \cdot e^{\frac{-2 \pi i}{N} \cdot j \cdot k} \quad \text { for } \mathrm{k}=0 \rightarrow \mathrm{N}-1 \quad \text { Equation } 3
$$

This transformation creates a series of $\mathrm{N} / 2$ distinct complex Fourier coefficients $A_{I_{d}}(k)$ where $k$ is an index referring to the discrete frequency $f(k)=k \cdot$ samplerate $/ N$ in Hz. Due to the Nyquist-Shannon sampling theorem the highest measured frequency in a signal is half that of the samplerate used to acquire the signal. In this experiment we will be primarily applying Fourier transforms to signals sampled at $100 \mathrm{kHz}$, so transient oscillations at frequencies $>50 \mathrm{kHz}$ will not be observed. HET plasmas are known to possess oscillations at higher frequencies [1], but the amplitudes of these oscillations are much less than the HET fundamental mode (i.e., the breathing mode), which for the BHT-600 thruster resides in the band $15 \mathrm{kHz}-45 \mathrm{kHz}$ (see Figure 1). The Power Spectral Density is defined from the Fourier coefficients as:

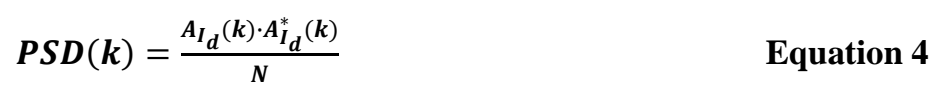

The PSD or power spectrum is real valued (not complex as the raw Fourier coefficients) and provides a measure of how the energy of a signal is distributed at different frequencies. A highly oscillatory system - such as an undamped spring mass system or an HET - exhibit a spike at their characteristic frequency of oscillation and possible harmonics at higher frequencies.

\section{F. Transfer Functions and System Modeling}

While PSD functions display the nature of the oscillations present in a signal, they do not provide any information about the system that generated the signal. With two different signals related to each other in some way, such as the output from two sensors located a fixed distance apart, one can construct a transfer function that models the relationship between the two signals. For this research project we shall model our Hall thruster and plume system, using the discharge current as the input signal and the plume electron density at a fixed $x-y$ coordinate as the output. The averaged transfer function between these two signals is then:

$$
\overline{\boldsymbol{H}}(\boldsymbol{x}, \boldsymbol{y}, \boldsymbol{f})=\frac{\mathbf{1}}{\boldsymbol{M}} \sum_{\boldsymbol{m}=\mathbf{1}}^{\boldsymbol{M}}\left[\frac{\mathcal{F}\left\{\boldsymbol{n}_{\boldsymbol{e}, \boldsymbol{m}}(\boldsymbol{x}, \boldsymbol{y}, \boldsymbol{t})\right\}}{\mathcal{F}\left\{\boldsymbol{I}_{\boldsymbol{d}, \boldsymbol{m}}(\boldsymbol{x}, \boldsymbol{y}, t)\right\}}\right], \quad \boldsymbol{M}=\text { sets to average (typically } 10 \text { to } 100 \text { sets) }
$$

\section{Equation 5}


This transfer function, in capturing the phase and magnitude differences between the $I_{d}(t)$ and $n_{e}(t)$ signals, models how discharge current transients give birth to electron density transients. By computing the transfer functions for a full grid of $\mathrm{x}-\mathrm{y}$ points within the plume of our thruster, we thereby create a matrix of transfer functions, that can be used to create a model for the entire plume of our HET. Thus, with a single discharge current input signal we will be able to recreate the electron density signals at all spatial $\mathrm{x}-\mathrm{y}$ locations. This technique allows for the creation of 2D animations (discussed later in Section $\mathrm{K}$ ) of the plume electron density transient fluctuations. It is important to point out that these system modeling transfer functions are not complete models of the thruster-plume system. Proper modeling of a system is termed "system identification" and typically involves the use of special input signals such as chirps or band-limited white noise. These special inputs excite the system with an equal amount of energy at each frequency, thereby facilitating modeling the system in a more complete sense. Since we cannot control the input signal to our HET-plume system we cannot perform a complete system identification. Thus, our model will only provide accurate modeling when the input signal is similar (i.e., has the same frequency response or PSD content) to the input signal initially used in the construction of our transfer function. However, by using sampled discharge current signals at the same thruster operating conditions, our modeling will be quite precise. While the transient oscillations in the discharge current and electron density are turbulent in nature, their spectral responses are time invariant. Thus, at the expense of gaining a coarser frequency resolution, averaging may be applied in the construction of our transfer functions by cutting up the signals into $\mathrm{M}$ pieces and obtaining a set of $\mathrm{M}$ transfer functions that can then be averaged together at each frequency. This process produces better statistical models that more accurately capture the behavior of the Hall thruster breathing mode transients. Unlike smoothing data in the time domain, we are not smearing out the data in the frequency domain. We are instead reducing the number of modeled frequencies by a factor of $M$ and reducing uncertainty by a factor of $\sqrt{M}$.

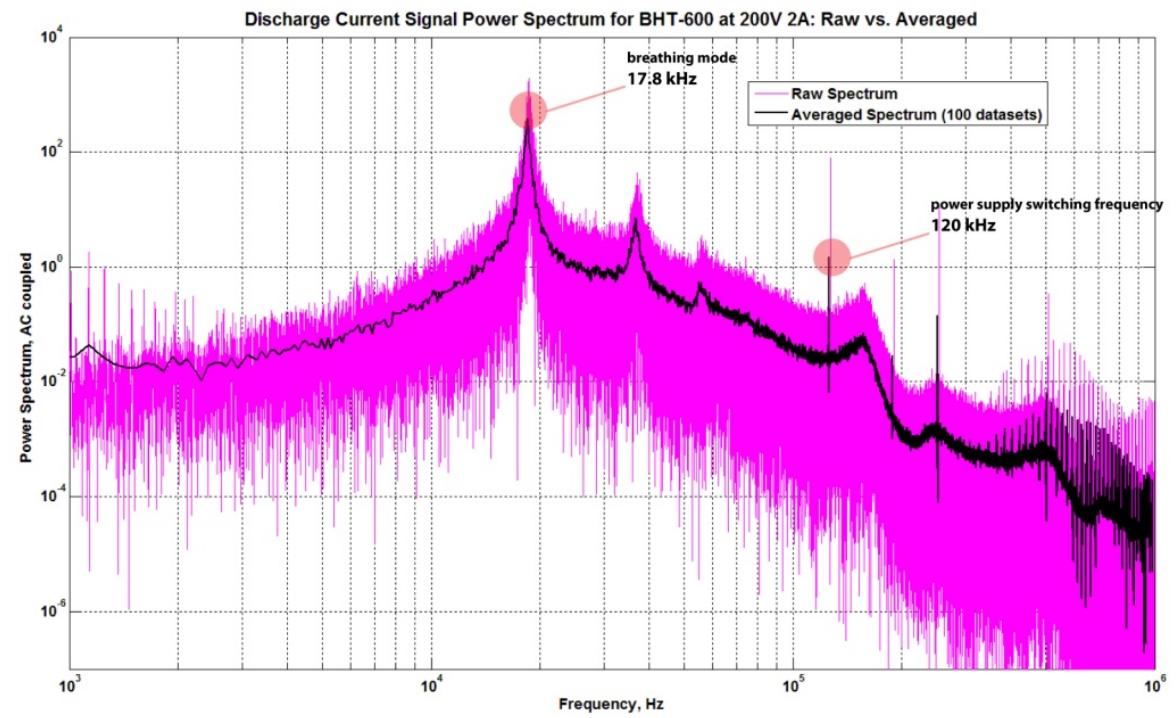

Figure 7. Effectiveness of averaging Fast Fourier Transform (FFT) coefficients as a means of enhancing statistical confidence of measured frequency content in turbulently fluctuating signals. In this plot we have included the power spectrum as measured in the discharge current signal for the frequency band $1 \mathrm{kHz}$ to $1 \mathrm{MHz}$. The primary mode at $17.8 \mathrm{kHz}$ is the thruster breathing mode. Many additional modes are apparent at frequencies above the breathing mode, but the magnitudes of these oscillations are orders of magnitude smaller than the thruster primary mode at $18 \mathrm{kHz}$.

Sharp peaks are not lost as a consequence of this averaging, and noting the very narrow spike at $120 \mathrm{kHz}$ (Figure 7) we see this peak in both the raw and 100-piece averaged version. The frequency of $120 \mathrm{kHz}$ corresponds to the thruster discharge power supply switching frequency. Only peaks narrower than the discrete $\Delta f$ frequency resolution $(\Delta f=100 \mathrm{~Hz}$ in averaged trace of Figure 7) are lost with averaging.

\section{G. Fourier Synthesis}

Converting a signal from the frequency domain into the time domain is referred to as Fourier synthesis. For a discrete collection of points the inverse Fourier transformation used to synthesize time domain data is defined as:

$$
\mathcal{F}^{-1}\left\{A_{I_{d}}(j)\right\}=I_{d}(\Delta t \cdot j)=\frac{1}{N} \sum_{k=0}^{N-1} A_{I_{d}}(k) \cdot e^{\frac{2 \pi i}{N} \cdot j \cdot k} \quad \text { for } \mathrm{j}=0 \rightarrow \mathrm{N}-1 \quad \text { Equation } 6
$$


In the preceding equation we have used the discharge current signal $I_{D}(t)$ and its Fourier coefficients $A_{I_{D}}(k)$. Comparing Equation 3 and Equation 6 we see the relative ease with which we can transfer a signal from the time domain to the frequency domain and then back to the time domain if so desired. The dualism inherent to Fourier analysis ensures that the synthesized signal will exactly match the initial time domain signal; mathematically:

$$
\mathcal{F}^{-1}\left\{\mathcal{F}\left\{I_{d}(t)\right\}\right\}=I_{d}(t) \quad \text { Equation } 7
$$

The ability to exactly recover the original signal is possible due to the lack of signal manipulation; i.e., filtering. If one does employ methods of signal processing to filter the frequency space response of the signal then the exact signal will not be recovered as in Equation 7. But moderate filtering techniques, such as the FFT coefficient averaging (see Figure 7), will create only minor differences between the synthesized time domain data and the original time domain data. The bulk of signal processing theory is based on this principle; relying on the use of frequency space filters to improve various characteristics of signals initially sampled in the time domain.
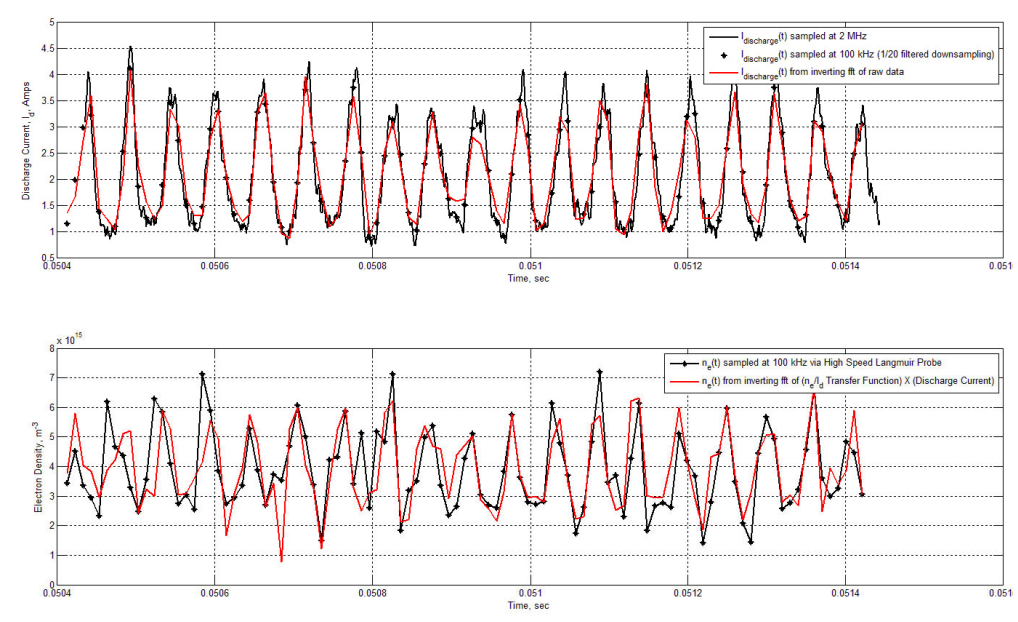

Figure 8. Synthesized time domain signals (in red) compared with the raw time domain signals (in black). Even with the transfer function and Fourier coefficient averaging, the synthesized time domain data closely matches the original signal.

In Figure 8 we see an example of synthesized thruster discharge current and electron density signals. The recreation is not exact, but quite close considering the amount of signal processing that occurs to obtain the synthesized data (see Figure 9). If we had not performed any signal processing the signals would be identical per Equation 7.

The true usefulness of the application of Fourier theory in interpreting the HET transient fluctuations is dramatically apparent in our ability to combine hundreds of distinct data-sets of plume transients into a single 2D spatial grid that is then animated at a 100,000 frames per second.

\section{H. Signal Processing}

Shown in Figure 9 is brief summary of the various steps applied to the enormous quantity of data collected. Using network communication, several PCs automate the motion of two motion control tables to position the Langmuir probe point-by-point across a pre-defined grid with appropriate delays for data collection and vibrational damping (total dwell time per point of $30 \mathrm{sec}$ ). At each grid-point, a command is sent to the high-speed ADI rtX data collection system to collect $1 \mathrm{sec}$ of data at $2 \mathrm{MHz}$. Once acquired these synchronously sampled analog-to-digital voltage signals, are then post processed to convert to the current and voltage signals they represent; applying voltage dividing factors, amplifier gains, and shunt constants. The current signal from the null probe is directly subtracted from the Langmuir probe signal and the resultant signal is chopped up into 100,000 distinct I-V traces each time-stamped at the midpoint of their acquisition. An automated Langmuir probe algorithm is then applied to interpret each I-V trace extracting the transient plasma properties of: electron density, electron temperature, plasma potential, and floating potential. While the signal processing could be concluded having attained the plasma transient behavior at $100 \mathrm{kHz}$, we go further and move our signals into the frequency domain with fast Fourier transforms. Then, for each grid-point, an averaged transfer function is formed between the electron density spectrum measured at each plume coordinate and the thruster discharge current spectrum sampled simultaneously. As discussed in Section F, this averaged transfer function provides a model of how the transient fluctuations in the discharge current setup corresponding fluctuations in the electron density, preserving the correct phase and magnitude of this turbulent phenomenon. After collecting a full matrix of these averaged transfer functions - one for each of 352 grid-points within the HET plume - we then pick a single '*' location to serve as our reference location. Taking the discharge current FFT coefficients at this '*' location as inputs to all transfer functions in our matrix we then create a matrix of Fourier coefficients for the electron density at all plume grid-points. Applying inverse Fourier transformations to this matrix of signals returns them to the time domain were they are all completely synchronized with each other due to the use of the single ' $*$ ' input signal used in their generation. Because we applied AC filtering to the initial time domain signals (and windowing in FFT preparation) these synthesized time-domain electron density signals are fluctuations about a zero valued mean. Thus, after de-windowing these signals we re-add the mean electron density to 
each signal. The map of mean densities is obtained by averaging the same transient signals (prior to windowing and AC filtering) at each plume coordinate (see also Figure 13). This reconstitutes the full magnitude of the transient electron density fluctuations for the full plume, in a fully time coherent manner.

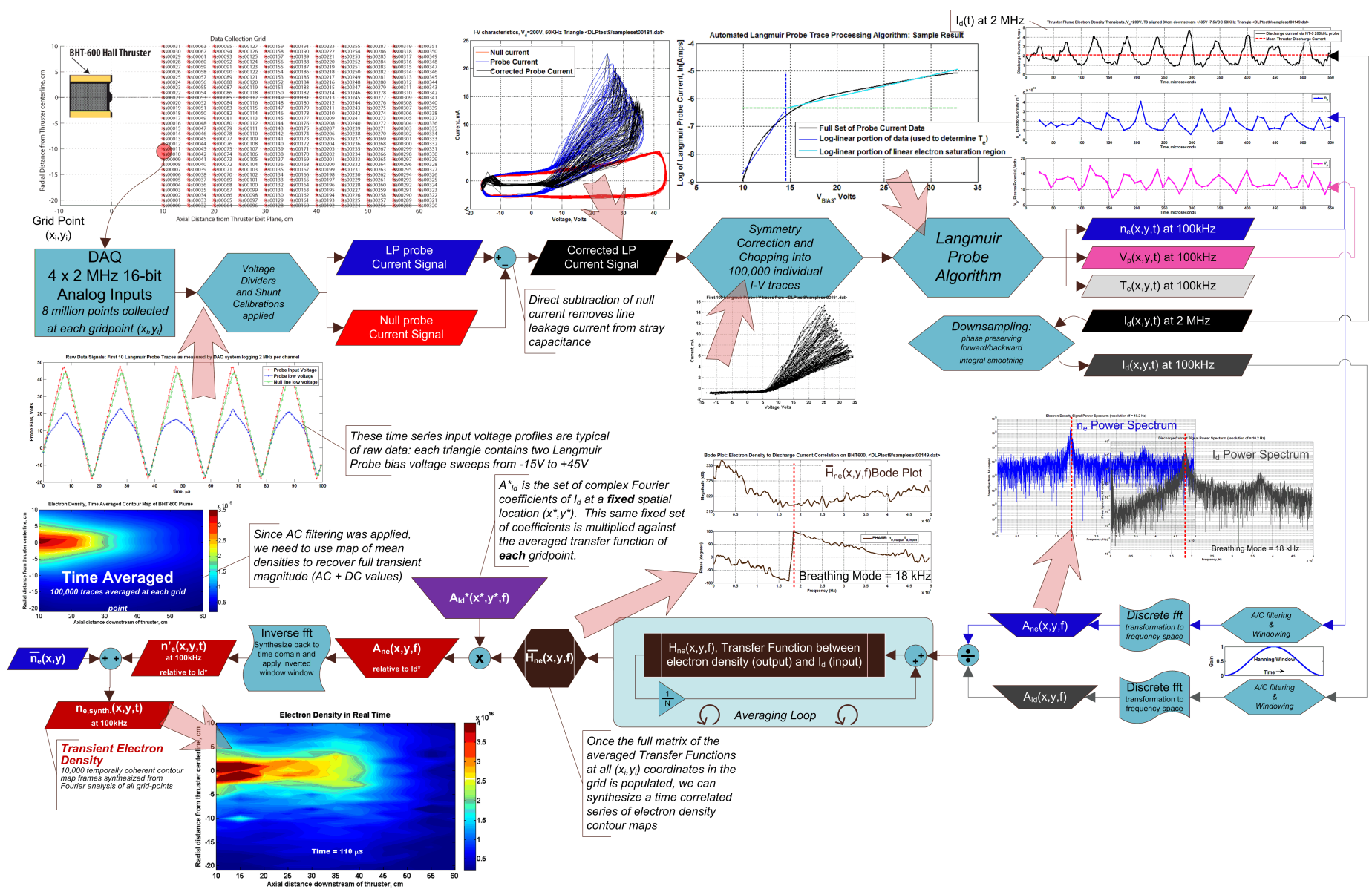

Figure 9. Summary of signal processing: progressing in clockwise manner, starting with raw data in upper left corner to transient plasma properties in upper right corner and then completing with synthesized full plume transient frames in lower left corner. Small plots representing real data are included at key steps to show a small portion of the signals.

\section{Results}

\section{Electron Density Transients}

At a single spatial location in the plume we sample the plasma properties 100,000 times over the course of one second of data acquisition. After processing the raw data as described, we obtain the plume transient: electron density, election temperature, floating potential, and plasma potential, along with the thruster discharge current signal. The first plasma signal we present, the transient electron density, is shown in Figure 10. While the discharge current is demonstrating the
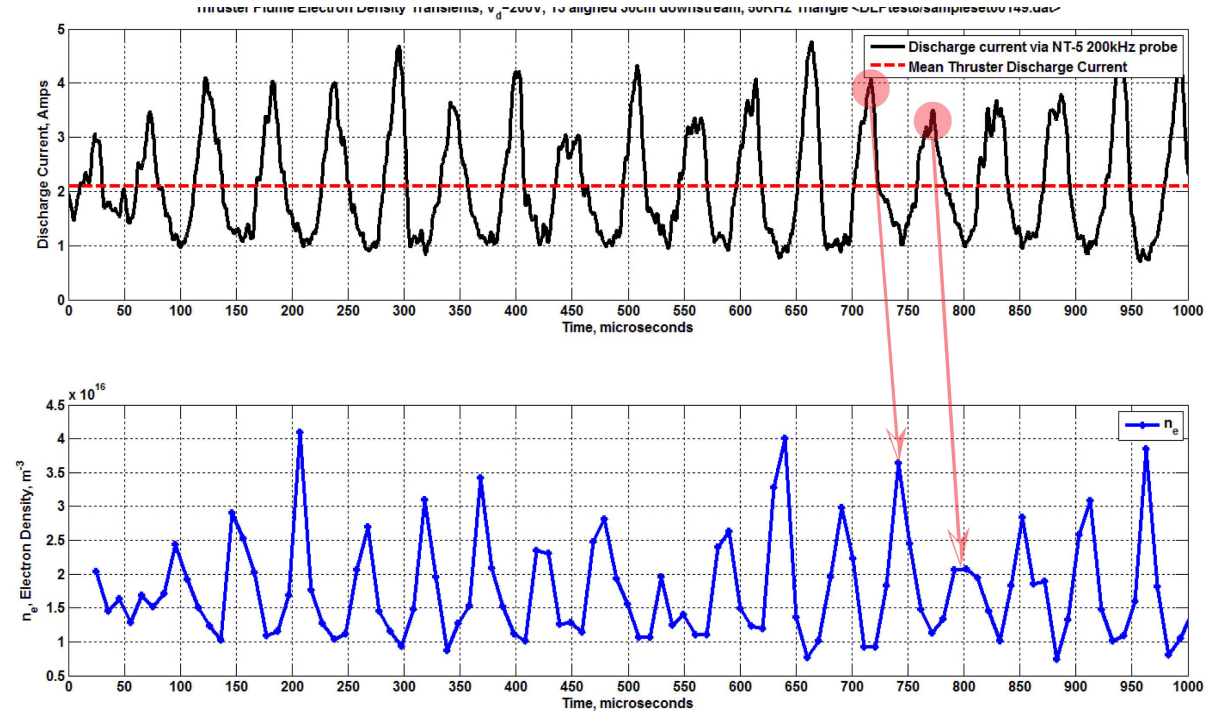

Figure 10. Electron density transient oscillations $30 \mathrm{~cm}$ downstream. Note the small phase delay $(27 \mu \mathrm{s})$ between peaks in the two signals, representing the ion transit time of $11 \mathrm{~km} / \mathrm{s}$. Also, notice the relative magnitudes of oscillation are nearly identical with the peak/trough ratios ranging from 3 to 4 .

American Institute of Aeronautics and Astronautics 092407 
expected behavior rich with large amplitude oscillations cycling between 1 and $4 \mathrm{~A}$, it is rather shocking to see nearly the same waveform in the electron density signal. As the electron density is fluctuating between $1 \times 10^{16}$ and $4 \times 10^{16} \mathrm{~m}^{-3}$, the relative magnitudes of these oscillations are roughly equivalent. The presence of breathing mode oscillations has been known to exist in the near field of Hall thrusters [1, 4, 9, 14], but at $30 \mathrm{~cm}$ downstream it appears these oscillations propagate relatively undamped through space.

\begin{tabular}{|c|c|c|c|c|}
\hline $\begin{array}{l}\text { Downstream Measurement } \\
\text { Position }\end{array}$ & $n_{e, p e a k-t o-p e a k}^{\prime}\left(m^{-3}\right)$ & $\overline{n_{e}}\left(m^{-3}\right)$ & $\frac{n_{e, p e a k-t o-p e a k}^{\prime}}{\overline{n_{e}}}$ & $\frac{I_{D, p e a k-t o-p e a k}^{\prime}}{\overline{I_{D}}}$ \\
\hline $15 \mathrm{~cm}$ & $1.8 \times 10^{16}$ & $2.3 \times 10^{16}$ & 0.76 & 1.07 \\
\hline $20 \mathrm{~cm}$ & $1.6 \times 10^{16}$ & $2.0 \times 10^{16}$ & 0.79 & 1.08 \\
\hline $30 \mathrm{~cm}$ & $1.3 \times 10^{16}$ & $1.6 \times 10^{16}$ & 0.80 & 1.08 \\
\hline $45 \mathrm{~cm}$ & $7.8 \times 10^{15}$ & $8.9 \times 10^{15}$ & 0.87 & 1.10 \\
\hline $60 \mathrm{~cm}$ & $4.3 \times 10^{15}$ & $5.3 \times 10^{15}$ & 0.81 & 1.11 \\
\hline
\end{tabular}

Table 1. Tabulation of transient fluctuation magnitudes, downstream, discharge channel aligned.

Using fluctuation magnitude notation - common to the study of turbulent flows - we collect the fluctuation properties from five different axial positions in Table 1. Specifically in our definition of the non-dimensional fluctuation magnitude as peak-topeak/mean values we thus have:

$$
\frac{n_{e, p e a k-t o-p e a k}^{\prime}}{\bar{n}_{e}} \approx 0.81, \text { Constant axially (channel aligned) }
$$

\section{Equation 8}

Equation 8 uses the peak-to-peak value of the fluctuation (AC) component of the electron density with the total transient $\left(\right.$ AC+DC) electron density expressed as: $n_{e}(t)=n_{e}^{\prime}(t)+\overline{n_{e}}$. Throughout the extent of this experiment we operated the BHT-600 thruster at a fixed 200 Volt, 2 Amp discharge, providing a nearly constant discharge current fluctuation magnitude:

$$
\frac{I_{D, p e a k-t o-p e a k}^{\prime}}{\bar{I}_{D}}=1.1, \text { Constant } 200 \mathrm{~V}, 2 \text { Adischarge } \quad \text { Equation } 9
$$

The fluctuation magnitudes of the electron density and discharge current signals are quite close which makes sense since they are both measuring the same phenomena. The pulses of plasma waves (as in Figure 12) set up by the thruster breathing mode ionization instability appear to traverse the interrogation region of the plume (axial: $10 \mathrm{~cm}$ to $60 \mathrm{~cm}$ ) completely undamped. Solution of the Euler equations in the zero pressure limit predicts a theoretical $1 / \mathrm{R}^{2}$ drop in density, as the jet exhaust disperses in the vacuum, and this drop appears equivalently in both the fluctuation and mean. This implies that the transients in electron density will propagate indefinitely through space until complete plume neutralization is achieved.

The key difference in the simultaneously sampled signals of Figure 10 is the phase lag in the electron density signal, which trails behind the discharge current signal. While one can simply measure the distance between peaks to measure this phase lag directly as roughly $27 \mu \mathrm{s}$, a more statically accurate method is to use the phase as measured in a the Bode plot of the transfer function between the two signals where we locate the phase measured at the breathing mode, $17.8 \mathrm{kHz}$, as $171.8^{\circ}$ (nearly opposite in phase). Thus, the transient propagation delay is statically estimated as:

$$
\tau_{\text {delay }}(30 \mathrm{~cm})=\frac{\phi_{\text {delay }}}{2 \pi} \cdot \frac{1}{f_{\text {breathing }}}=\frac{172^{\circ}}{360^{\circ}} \cdot \frac{1}{17800 \mathrm{~Hz}}=26.8 \mu \mathrm{s} \quad \text { Equation } 10
$$

The carriers of these transient oscillations are waves of ions generated in the pulses of the thruster breathing; a fact supported when the theoretical ion transit time is compared to this value. Assuming the singly charged xenon propellant ions are fully accelerated at the thruster exit plane, we estimate the following theoretical transit time:

$$
\tau_{\text {ion transit time }}(30 \mathrm{~cm})=\frac{L}{\sqrt{\frac{2 V_{d} \eta_{d}}{m_{\text {ion }}}}}=(0.3 \mathrm{~m}) \sqrt{\frac{2.2 \times 10^{-25} \mathrm{~kg}}{2\left(1.6 \times 10^{-19} \mathrm{C}\right) \cdot(200 \mathrm{~V}) \cdot(0.80)}}=19.7 \mu \mathrm{s}
$$




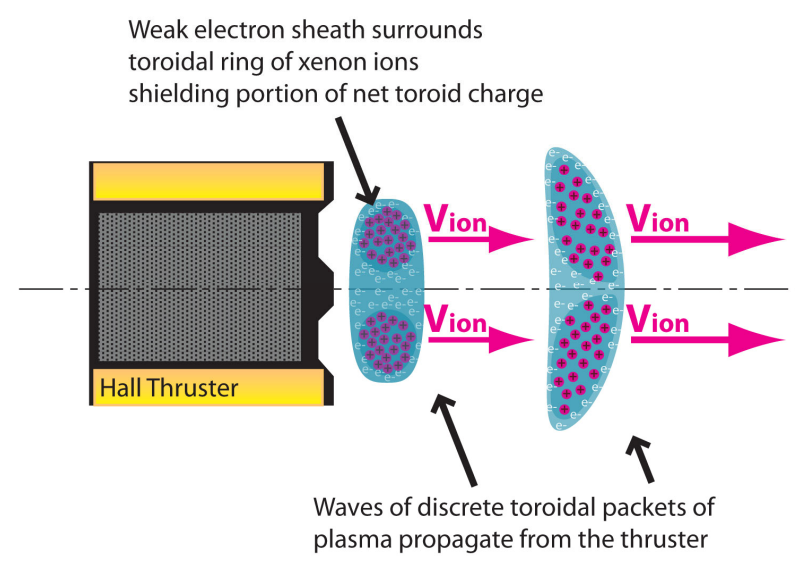

Figure 12. Waves of relatively dense plasma are emitted as the thruster "exhales" ions during each breathing mode oscillation. Partial charge shielding of the bulk donut shaped ion mass may limit the electric field acceleration force.

established by the anode discharge voltage. The regions of space between the dense plasma structures are relatively devoid of plasma containing less than $1 / 4^{\text {th }}$ the density. Since the bulk of the thruster propellant $(>75 \%)$ is contained within these toroidal breathes of plasma, their axial velocity ( $V_{\text {ion }}$ in Figure 12 ) is representative of the time averaged propellant particle velocity.

The assumption of complete acceleration at the exit plane is known to be incorrect [22-24], and with the acceleration zone extending into the plume, an under-estimate of the ion transit time is obtained by Equation 11. Additional acceleration is occurring in the plume downstream from the discharge channel exit plane. This effect accounts for a portion of the difference in the measured ion speed from Equation 10. Taking the ion speed as the toroid speed measured, we have that, $v_{\text {ion }}=\frac{0.30 \mathrm{~m}}{26.8 \times 10^{-6} \mathrm{~s}}=$ $11,190 \frac{\mathrm{m}}{\mathrm{s}}$ or $11.2 \frac{\mathrm{km}}{\mathrm{s}}$, With this we can compute a measure of the thruster total specific impulse at this downstream position:

$$
I_{s p, 30 \mathrm{~cm}} \equiv \frac{v_{\text {propellant }}^{*}}{g_{0}} \cong \frac{v_{\text {ion }}}{g_{0}}=\frac{11.2 \times 10^{3} \mathrm{~ms}^{-1}}{9.81 \mathrm{~ms}^{-2}}=1141 \mathrm{sec}
$$

\section{Equation 12}

In performance measurements with this same thruster at very similar operating conditions (200V 3A discharge) [18], the total specific impulse was measured as $\mathbf{1 2 7 1} \mathbf{~ s e c ~} \pm \mathbf{2 8} \mathbf{~ s e c}$, which only differs by $10 \%$. Using a position further downstream, after the ions have completed their acceleration, with Equation 12, we find $I_{s p, \infty}=1260 \mathrm{sec}$, which matches nearly perfectly $(<1 \%$ difference) to our independent performance measurement of the thruster specific impulse.

It should be noticed that the signal for the transient electron density is relatively noise free, in that the only fluctuations appear to be real plasma fluctuations since they are well mirrored in the discharge current signal. The reason for this is that the electron density is directly proportional to the electron saturation current, $I_{\text {sat,e }}$. The custom current sensing circuitry developed for this research provided a signal-to-noise ratio of $80 \mathrm{~dB}$, effectively ensuring a low-noise floor in our $I_{\text {sat,e }}(t)$ signal. The same fluctuations also appear in the $T_{e}, V_{f}$, and $V_{p}$ signals measured by our high-speed Langmuir probe system, but these signals suffer from additional noise due to sensitivity arising from the method of $\mathrm{I}-\mathrm{V}$ characteristic analysis applied. Ongoing work is in progress to increase the confidence in these parameters computed by the automated Langmuir probe data processing algorithm. 

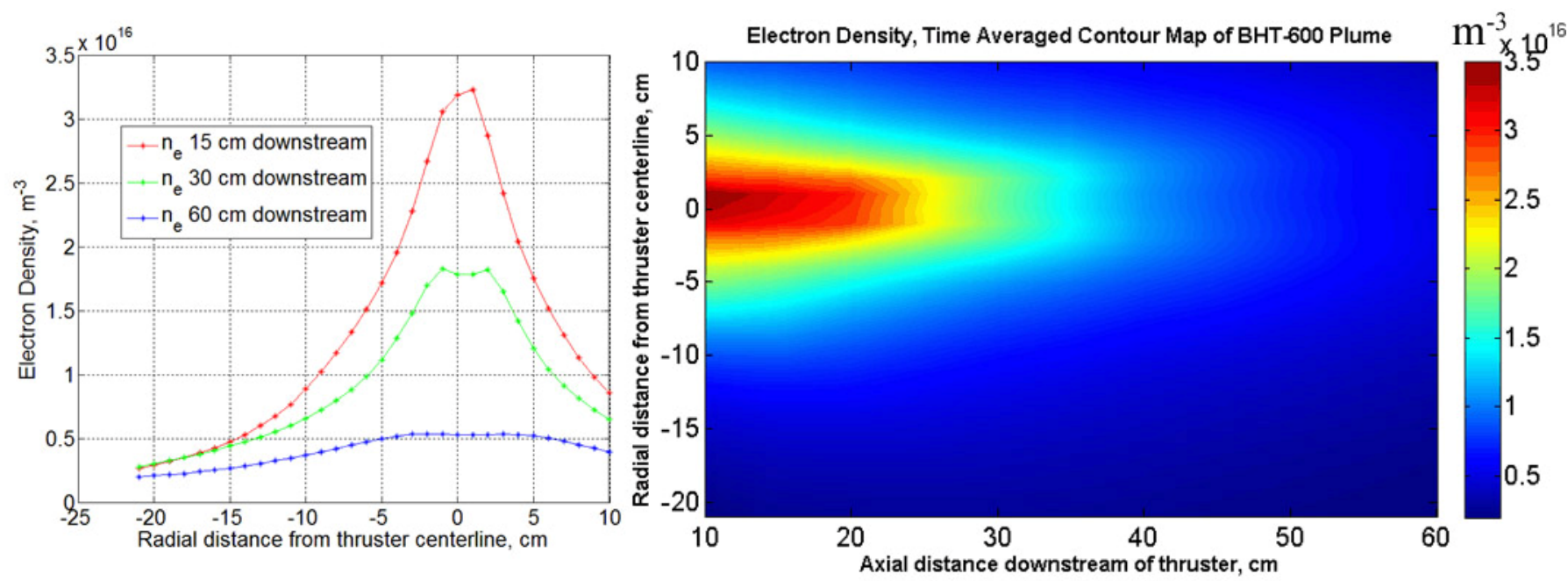

Figure 13. Time-averaged electron density contour map. The plasma density collected at $100 \mathrm{kHz}$ is time-averaged to produce the above profiles and the contour map.

\section{J. Power Spectrums}

Large multi-million sample-point signals were acquired in the time domain so that clear power spectral plots and cross-signal transfer functions could be acquired. Application of Fourier transformations and the other formulas presented, allows for evaluation of the spectral content of each transient signal. Combining all of the signals into a single plot we have the following (see Figure 14):

Power spectral density plots are very useful tools in examining the frequency contents of various signals. The power spectral traces in Figure 14 show that in addition to the breathing mode at $17.8 \mathrm{kHz}$, there is a harmonic of this mode at

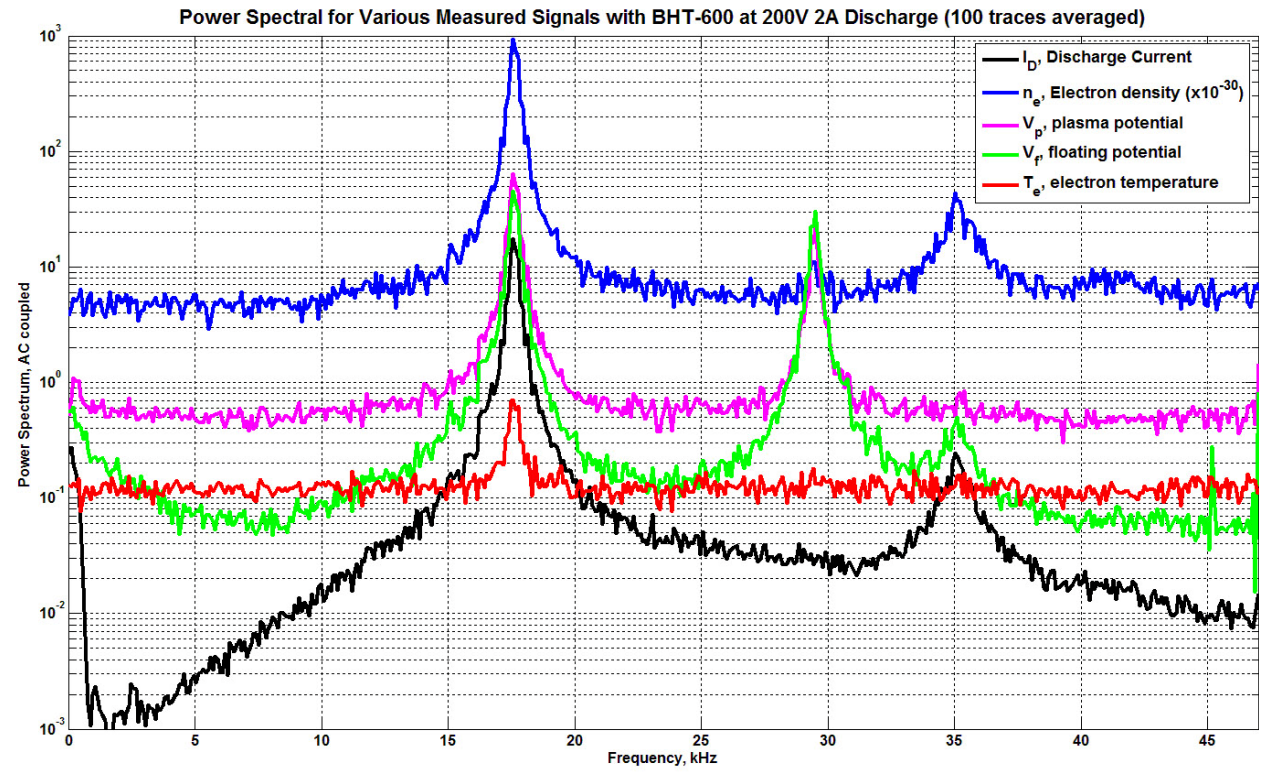

Figure 14. Spectral nature of transient plume fluctuations. These signals were acquired $30 \mathrm{~cm}$ downstream of the BHT-600 Hall thruster. The breathing mode frequency, $17.8 \mathrm{kHz}$, is the dominant feature in each signal. $35 \mathrm{kHz}$, which is common for undamped oscillatory processes such as the HET breathing mode phenomena. One other mode appears quite strongly in the transient plasma potential and floating potential signals, at $28 \mathrm{kHz}$. The origin of this mode is not known, but may arise from geometry effects, cathode coupling instabilities, or charge-exchange processes. Since this mode is almost non-existent in the discharge current signal it is caused by a process that is plume ion specific; i.e., lacking backwards communication towards the thruster.

\section{K. Plume Transient 2D Animations}

Exploiting the rich repository of relative phase and magnitude information stored in a matrix of transfer functions between two signals, we may synthesize another matrix of the original time domain signals related to one another in having the same input signal. This common input allows us to track two-dimensional waves of plasma density fluctuations as they propagate downstream from the Hall thruster, as demonstrated in Figure 15. 


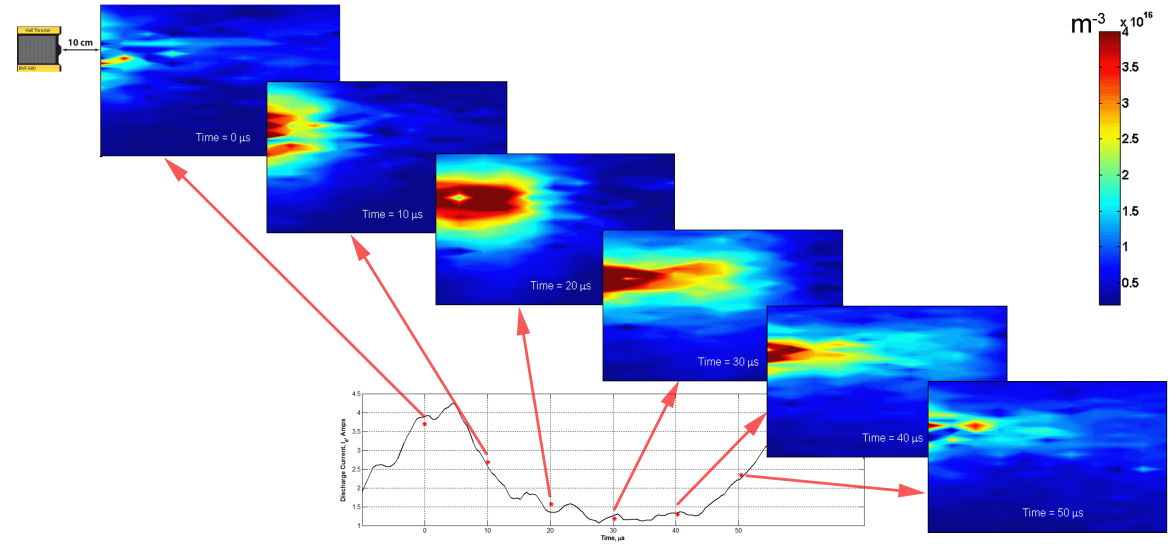

Figure 15. 2D frames of transient plume total electron density at 100,000 frames per second. The input signal used to generate these time-coherent electron density contour maps is the discharge current signal shown in the lower left. The red stars indicate the actual current values used since we needed to match all the signal sample rates to create our discrete time Transfer function matrix. Note: each frame measures $31 \mathrm{~cm}$ (radial) by $50 \mathrm{~cm}$ (axial) with the left edges $10 \mathrm{~cm}$ downstream from the HET channel exit plane. fluctuation magnitude within the entire $(0.31 \times 0.5 \mathrm{~m})$ plume region examined. To see this we next show the exact same data frames but instead of plotting the total electron density in $\mathrm{m}^{-3}$, we plot the non-dimensional instantaneous (not peak-to-peak) fluctuation magnitude of the electron density:

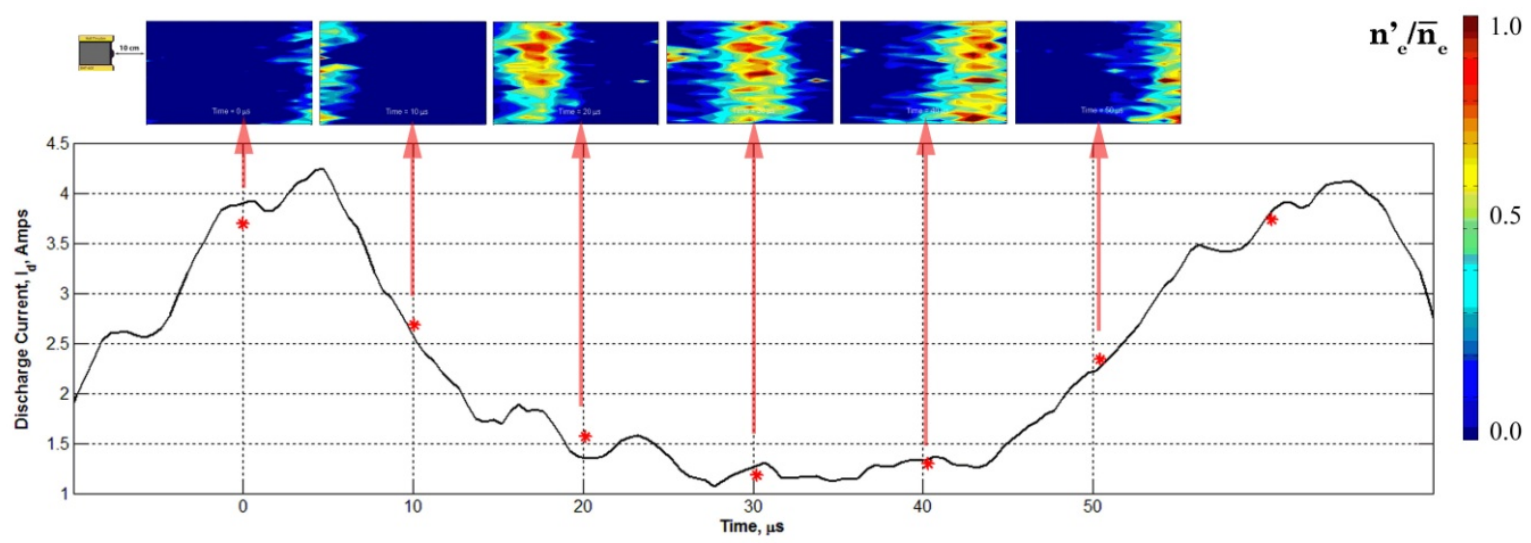

Figure 16. Non-dimensional localized electron density instantaneous fluctuation magnitude for visualization of plasma waves. By plotting the fluctuation magnitude relative to the average density, we remove the $1 / \mathrm{R}^{2}$ decay in the visible plasma transients. Note: the color bar for the density contour map frames is scaled so that: blue $=0$, yellow $=0.6$, and red $=1$. Also note that the same data are shown in this and the prior figures.

By plotting the localized electron density fluctuation magnitude we remove the $1 / \mathrm{R}^{2}$ decay both axially and radially. The resulting waves of relatively dense plasma fluctuations travel away from the thruster un-dissipated; as was hypothesized in Figure 12. Again, the simultaneously sampled discharge current has been included to show the temporal lag of the density wave. The relative magnitude for the wave seems to remain constant as $\frac{\overline{\left.n_{e}^{\prime}(t)\right|_{\text {local-max }}}-\overline{\left.n_{e}^{\prime}(t)\right|_{\text {local-min }}}}{\overline{n_{e}}}=\frac{n_{e, p e a k-t o-\text { peak }}^{\prime}}{\overline{n_{e}}} \approx 0.81$. Plotting density in this manner introduces a significant amount of noise since the denominator is quite small in the far field, thus amplifying the signal noise and underlying plasma turbulence. Even so, the dark blue regions in Figure 16 emphasize the large magnitude (red and yellow regions) of the plasma wave emitted by the Hall thruster breathing mode. An illustration of the BHT600 Hall thruster is included in both Figure 15 and Figure 16 - drawn close to actual scale - to show the position of the thruster relative to the plume region mapped in each of the electron density contour map frames. Approximately one cycle of the breathing mode oscillation, $\Delta t_{\text {cycle }}=1 /(17800 \mathrm{~Hz})=56 \mu \mathrm{s}$, is shown in both Figure 15 and Figure 16. As our final result, we plot a 3D representation of the transient fluctuations in Figure 17. We identify the radial profile as having a narrow Gaussian shape, and by adding the additional axis for time, a clear set of waves are seen to propagate past this axially fixed strip at a rate of 
$17.8 \mathrm{kHz}$ - one wave for each "breath" of xenon the thruster draws. Behind the four Himalayan mountains of density shown in Figure 17, the corresponding temporal discharge current trace is shown on an identical and aligned time axis. A short (ion transit time) delay of about 25- $\mu$ s is evident when comparing the $I_{d}(t)$ peaks to the resultant $n_{e}(t)$ peaks at this location $30 \mathrm{~cm}$ downstream from the BHT-600 exit plane suggesting an ion speed of nearly $12 \mathrm{~km} / \mathrm{sec}$.

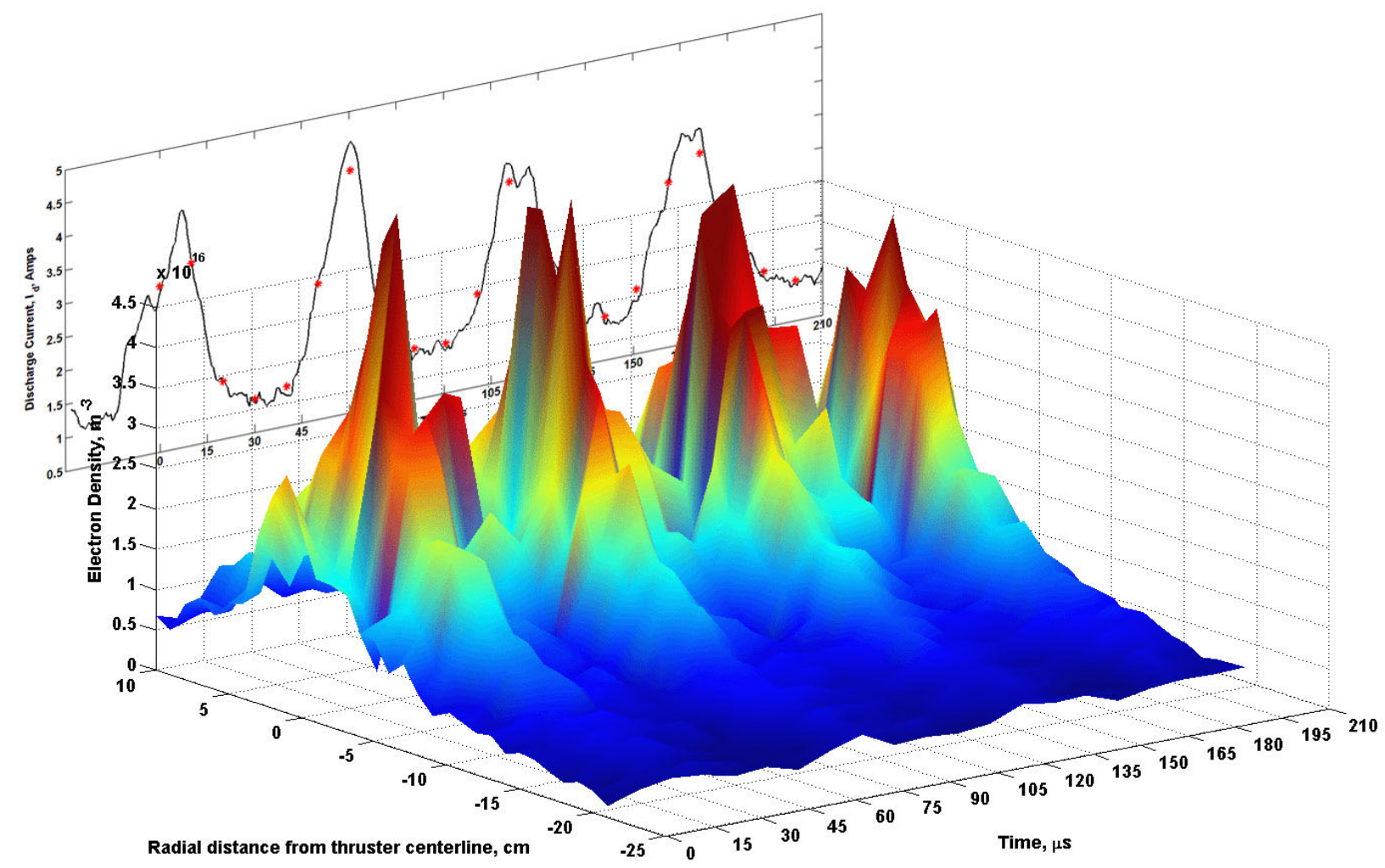

Figure 17. 3D plot $\left(\mathrm{z}=\boldsymbol{n}_{\boldsymbol{e}}, \boldsymbol{y}=\right.$ radial-position, $x=$ time $)$ of radial profile transient plasma density waves. In this surface plot of electron density we display the temporal variation of a fixed radial profile. On the rear left wall the time aligned (matched $x$-axes) or synchronous discharge current signal is overlaid. The axial distance to the thruster is $30 \mathrm{~cm}$ here and the roughly $25-\mu \mathrm{s}$ flight time of ions to reach this profile introduces about $180^{\circ}$ of phase difference.

The electron density oscillations in Figures 15-17 occur indefinitely with continued discharge from the Hall thruster, and while these oscillations appear unstable, they are normal for the operation of this and all other Hall effect thrusters (though the magnitudes differ). These density fluctuations together with plasma potential and electron temperature fluctuations tell us that the flow field of a Hall thruster plume is far from quiet. Consideration of the enormous field gradients set up by these plume fluctuations and the various polarization and other particle drifts thus effected will surely further our understanding of Hall thruster physics enabling better modeling and ultimately, improved thruster design.

\section{Conclusion}

A means of measuring transient phenomena within the plume of a Hall effect thruster was realized with a temporal resolution of $10 \mu$ s. Standard thin sheath Langmuir probe theory was applied to the acquired high-speed data extracting the transient plasma properties of density, temperature and plasma and floating potential. Measurable delays between the signal of electron density and thruster discharge current correspond well to expected ion transit times. The transient electron temperature and plasma potential (and floating voltage) signals mirror the disruptions in discharge current in the same manner as the density but with no measurable phase lag - suggesting these are electron driven and electromagnetic field driven. The relative magnitude of the 17.8 $\mathrm{kHz}$ "breathing mode" type density fluctuations is nearly constant throughout the entire $0.3 \times 0.5$ meter region of the plume investigated: $n_{e}^{\prime} / \overline{n_{e}} \approx 0.81$. Fourier analysis was applied to the measured transient signals, and the spectral character of the plasma transient nature was dominated by the thruster breathing mode ionization instability. A complete set of transfer functions - one for each grid-point sampled in the plume - was used to create a statistically accurate model of the thruster-plume system. Using a common input signal of discharge current, we then successfully synthesized time-coherent signals at each of the 352 grid- 
points evaluated. Animations of electron density fluctuations at a framerate of $10 \mu$ s reveal waves of dense plasma emanating from the thruster - clearly visualizing the thruster breathing mode that dominates HET operation.

\section{References}

1. $\quad$ Choueiri, E.Y., Plasma oscillations in Hall thrusters. Physics of Plasmas, 2001. 8(4): p. 1411-1426.

2. Choueiri, E.Y., Characterization of oscillations in closed drift thrusters. 1994 30th AIAA/ASME/SAE/ASEE Joint Propulsion Conference; Indianapolis, IN; June 27-29, 1994, 1994.

3. Gascon, N., et al., Signal processing and non-linear behavior of a Stationary Plasma Thruster - First results. AIAA/ASME/SAE/ASEE Joint Propulsion Conference and Exhibit, 35th, Los Angeles, CA; 20-24 June 1999, 1999.

4. $\quad$ Chesta, E., et al., A characterization of plasma fluctuations within a Hall discharge. IEEE Transactions on Plasma Science, 2001. 29(4): p. 582-91.

5. Litvak, A.A., Y. Raitses, and N.J. Fisch, Experimental studies of high-frequency azimuthal waves in Hall thrusters. 4, 2004.

6. Baranov, V.I., et al., Theory of oscillations and conductivity for Hall thruster. AIAA, ASME, SAE, and ASEE, Joint Propulsion Conference and Exhibit, 32nd, Lake Buena Vista, FL; 1-3 July 1996, 1996.

7. $\quad$ Chesta, E., N.B. Meezan, and M.A. Cappelli, Stability of a magnetized Hall plasma discharge. 6, 2001.

8. $\quad$ Fife, J.M., M. Martinez-Sanchez, and J. Szabo. A numerical study of low-frequency discharge oscillations in Hall thrusters. in 33rd AIAA/ASME/SAE/ASEE Joint Propulsion Conference \& Exhibit. 1997. Seattle, WA.

9. Albarede, L., et al., Low-frequency electron dynamics in the near field of a Hall effect thruster. Physics of Plasmas, 2006. 13(6).

10. Kurzyna, J., et al., Spectral analysis of Hall-effect thruster plasma oscillations based on the empirical mode decomposition. 12, 2005.

11. Bradley, J.W., et al., Space and time resolved Langmuir probe measurements in a $100 \mathrm{kHz}$ pulsed rectangular magnetron system. Surface and Coatings Technology, 2001. 142-144: p. 337-341.

12. Chiodini, G., C. Riccardi, and M. Fontanesi, A $400 \mathrm{kHz}$, fast-sweep Langmuir probe for measuring plasma fluctuations. Review of Scientific Instruments, 1999. 70(6): p. 2681-2688.

13. Kim, Y.-W., et al., Time-resolved plasma measurement in a high-power pulsed ICP source for large area. Surface and Coatings Technology, 2004. 186(1-2): p. 161-164.

14. Lazurenko, A., et al., High-frequency instabilities and low-frequency dynamics in Hall thruster plasma. 40th AIAA/ASME/SAE/ASEE Joint Propulsion Conference and Exhibit; Fort Lauderdale, FL; July 11-14, 2004.

15. Riccardi, C., et al. Comparison between fast-sweep Langmuir probe and triple probe for fluctuations measurements. 2001: AIP.

16. Rudakov, D.L., et al. Fast electron temperature diagnostic based on Langmuir probe current harmonic detection on DIII-D. 2001: AIP.

17. Stranak, V., et al., Investigation of the time evolution of plasma parameters in a pulsed magnetron discharge. Czechoslovak Journal of Physics, 2006. 56: p. B1364-B1370.

18. Lobbia, R.B., Gallimore, Alec D., Performance Measurements from a Cluster of Four Hall Thrusters. 30th International Electric Propulsion Conference, Florence, Italy 2007. IEPC-2007-177.

19. Hargus Jr, W. and B. Pote, Examination of a Hall thruster start transient, in 38th AIAA/ASME/SAE/ASEE Joint Propulsion Conference and Exhibit. 2002: Indianapolis, IN.

20. A. V. Nedospasov, D.A.U., On Dynamical Effects on I-V Characteristic of Langmuir Probes in Tokamak Edge Plasma. Contributions to Plasma Physics, 1994. 34(2-3): p. 478-483.

21. I. Djermanov, N.D., Zh. Kiss'ovski and Ts. Tsankov, Langmuir probe differential measurement technique in inductively coupled RF plasmas. Journal of Physics: Conference Series 63 (2007) 012030, 2006. Second International Workshop \& Summer School on Plasma Physics 2006.

22. Haas, J.M. and A.D. Gallimore, Internal plasma potential profiles in a laboratory-model Hall thruster. 2001.

23. Hargus, W.A. and C.S. Charles, Near exit plane velocity field of a 200-Watt Hall thruster. Journal of Propulsion and Power, 2008. 24(1): p. 127-133.

24. Niemela, C.S., et al., Comparison of hall thruster plume expansion model with experimental data. STAR, 2006. 